\title{
Maximum entropy analysis of lattice QCD correlation functions
}

\section{Tom Harsono* and Frank X. Lee}

Department of Physics, The George Washington University, Washington, DC 20052, USA

E-Mail: harsono@gwmail.gwu .edu

The application of the Maximum Entropy Method (MEM) to the analysis of lattice QCD correlation function is discussed. We obtain information on the mass spectrum of excited states of a number of hadrons by extracting hadronic spectral functions (SPF) from correlation functions. Tests, results, and limitations of the MEM algorithm are presented. The data sets are on lattices $20^{3} \times 32$ covering over 10 meson states and 20 baryon states, with each state as a function of 26 quark masses.

The XXVI International Symposium on Lattice Field Theory

July 14 - 192008

Williamsburg, Virginia, USA

\footnotetext{
${ }^{*}$ Speaker.
} 


\section{Introduction}

Quantum Monte Carlo simulations of lattice QCD yield correlation functions (lattice QCD data) $D(\tau)$ as a function of imaginary time $\tau=\mathrm{i} t$. The real frequency spectral functions (SPFs) are then extracted from the correlation functions since SPFs of QCD contain physical information of hadrons such as masses, decay rates, etc. In this paper, we used MEM to extract ground and first excited states of some hadrons. MEM has been showed to be able to extract hadronic excited states with considerable success $[1,2,3]$. Zero temperature quenched lattice QCD data with overlap fermion are used in this study.

\section{MEM}

We follow Asakawa, et al. [2] in briefly outlining MEM as they already discussed it in detail. This method adopts Bryan's method [2,4], which is thought to be the state-of-the-art MEM. The relationship between data $D(\tau)$ and $\operatorname{SPF} A(\omega)$ at zero momentum is,

$$
D(\tau)=\int_{0}^{\infty} \mathrm{d} \omega A(\omega) K(\tau, \omega)
$$

where $K(\tau, \omega)$ is the kernel of the case in study. This is an inverse problem since we seek $A(\omega)$ for given $D(\tau)$. The method in extracting $A(\omega)$ is outlined below.

\subsection{Method}

Basicaly what MEM does is infer the most probable image $A$ from a given data $D$. The theoretical basis for MEM is Bayes' theorem in probability

$$
P[X \mid Y]=\frac{P[Y \mid X] P[X]}{P[Y]}
$$

where $P[X \mid Y]$ is the conditional probability of $X$ given $Y$. Let us rewrite the above in terms of our variables: let $D$ be the lattice QCD data and $H$ be the prior knowledge, e.g., $A(\omega \geq 0) \geq 0$. The conditional probability (also called the posterior probability) of SPF $A(\omega)$ given the data $D$ and prior knowledge $H$ is then

$$
P[A \mid D H]=\frac{P[D \mid A H] P[A \mid H]}{P[D \mid H]}
$$

$P[D \mid A H]$ is called the likelihood function[1]

$$
P[D \mid A H]=\frac{1}{Z_{L}} \mathrm{e}^{-L}
$$

where $L=\frac{1}{2} \chi^{2} \cdot P[D \mid H]$ a normalization constant independent of $A$. To maximize the posterior probability $P[A \mid D H]$, two independent parameters $\alpha$ and $m$ are inserted into the prior probability $P[A \mid H]$

$$
P[A \mid H] \rightarrow P[A \mid H \alpha m]=\frac{1}{Z_{S}} \mathrm{e}^{\alpha S}
$$


where $S$ is the Shannon-Jaynes entropy

$$
S[A]=\int_{0}^{\infty}\left[A(\omega)-m(\omega)-A(\omega) \log \left(\frac{A(\omega)}{m(\omega)}\right)\right] \mathrm{d} \omega \rightarrow \sum_{i=1}^{N_{\omega}}\left[A_{i}-m_{i}-A_{i} \log \left(\frac{A_{i}}{m_{i}}\right)\right]
$$

where $N_{\omega} \sim 10^{3}$ is the number of pixels into which the frequency is equally discretized to $\Delta \omega$. We look for a solution $A$ that maximizes the posterior probability

$$
P[A \mid D H \alpha m] \propto \mathrm{e}^{Q(A)}, \quad Q(A) \equiv \alpha S-L .
$$

Real and positive function $m(\omega)$ called the default model is introduced into the prior probability. The intermediary parameter $\alpha$ controls the relative weight of the entropy $S$, which pulls $A$ to fit the default model $m$ ), and it will be integrated out in the end. The likelihood function $L$ on the other hand pulls $A$ to fit the lattice data $D$. The default model used is $m \equiv m_{0} \omega^{n}(\mathrm{n}=2(5)$ for meson (baryon)). The parameter $m_{0}$ may be determined either by the perturbative asymptotic behavior of SPF at large $\omega, m(\omega \rightarrow$ large $)$, or by requiring that the resultant image $A_{\text {out }}$ has the least error.

MEM is able to reconstruct $A(\omega)$ and determine its statistical error. The error of $A(\omega)_{\text {out }}$ is averaged over an interval $I=\left[\omega_{\min }, \omega_{\max }\right]$. The average of $A$ for a given $\alpha$ over interval $I$ is defined as

$$
\left\langle A_{\alpha}\right\rangle_{I} \equiv \frac{\int[\mathrm{d} A] \int_{I} \mathrm{~d} \omega A(\omega) P[A \mid D H \alpha m] W(\omega)}{\int_{I} \mathrm{~d} \omega W(\omega)} \simeq \frac{\int_{I} \mathrm{~d} \omega A_{\alpha}(\omega) W(\omega)}{\int_{I} \mathrm{~d} \omega W(\omega)}=\frac{\int_{I} \mathrm{~d} \omega A_{\alpha}(\omega)}{\int_{I} \mathrm{~d} \omega}
$$

where $W(\omega)$ is a weight function and taken to be unity. It is also assumed that the posterior probability $P[A \mid D H \alpha m]$ is highly peaked around $A_{\alpha}(\omega)$ which is true for good data. This fact is also used to approximate the variance of $\left\langle A_{\alpha}\right\rangle_{I}, \delta A(\omega)=A(\omega)-A_{\alpha}(\omega)$, as

$$
\begin{aligned}
\left\langle\left(\delta A_{\alpha}\right)^{2}\right\rangle_{I} & =\frac{\int[\mathrm{d} A] \int_{I \times I} \mathrm{~d} \omega \mathrm{d} \omega^{\prime} \delta A(\omega) \delta A\left(\omega^{\prime}\right) P[A \mid D H \alpha m]}{\int_{I \times I} \mathrm{~d} \omega \mathrm{d} \omega^{\prime}} \\
& \simeq \frac{-\int_{I \times I} \mathrm{~d} \omega \mathrm{d} \omega^{\prime}\left(\frac{\delta^{2} Q}{\delta A(\omega) \delta A\left(\omega^{\prime}\right)}\right)_{A=A_{\alpha}}^{-1}}{\int_{I \times I} \mathrm{~d} \omega \mathrm{d} \omega^{\prime}}
\end{aligned}
$$

Therefore the error for $A_{\text {out }}$ in the region $I$ is given by

$$
\left\langle\left(\delta A_{\text {out }}\right)^{2}\right\rangle_{I} \equiv \int \mathrm{d} \alpha\left\langle\left(\delta A_{\alpha}\right)^{2}\right\rangle_{I} P[\alpha \mid D H m]
$$

which is shown as vertical bars in Fig. 2, 3, 4, and 5.

\subsection{Testing MEM}

Mock data were used to test how well MEM could produce sharp peaks and continuum given different quality of data. Our test results [5] agree very closely with Asakawa et al. [2]. Due to the limitation of space, only one test is presented here. As an example, we extracted $A_{\text {out }}(\omega)=$ $\omega^{2} \rho_{\text {out }}(\omega)$ from mock data, which consist of three Gaussian peaks of different widths. Figure 1 shows how well MEM works when the data get less noisy. Parameter $b$ adjusts the noise level of the mock data and $N$ is the number of time slices $(N=30$.) It is clear that as noise increases, the output image quality deteriorates as evidenced by the decreasing number of peaks reproduced and 

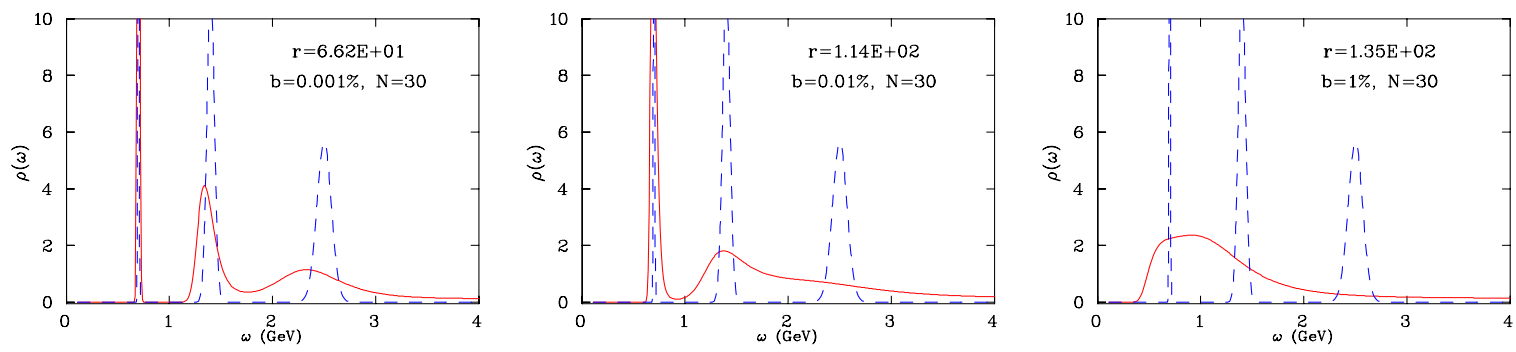

Figure 1: Test of MEM sensitivity to noise. $b$ is the noise level, $N$ is the number of temporal data points, and $r=\sum_{i=1}^{N_{\omega}}\left[\rho_{\text {out }}\left(\omega_{i}\right)-\rho_{\text {in }}\left(\omega_{i}\right)\right]^{2}$ is defined as the distance between $\rho_{\text {in }}$ and $\rho_{\text {out }}$ to measure how well the output matches the input. $\rho_{\text {in }}$ is dashed blue and $\rho_{\text {out }}$ is solid red. Left: All 3 peaks are reproduced. Middle: 2 out of 3 peaks are reproduced. Right: Only 1 broad peak is visible

the increasing valur of $r$. Noise turns out to be the most dominant factor influencing the quality of the output SPF. The more noisy the data get, the less information MEM produces to a point of unreliability. The next factors are the number of temporal data points $N$ and lattice spacing $a$ which are discussed in Ref. [2]. Good quality data with low noise are crucial in getting reliable results from MEM.

\section{Results and discussion}

The data we analyze are obtained from the Iwasaki gauge action and overlap fermion action [6] in the $\chi \mathrm{QCD}$ collaboration [7]. All data (except for pion) are of size $20^{3} \times 32$ with lattice spacing $a \sim 0.17 \mathrm{fm}$ and large lattice size $L a=3.4 \mathrm{fm}(\beta=6.0$ and $\kappa=0.1530)$. The number of gauge configurations is $N_{\text {conf }}=110$. The data for pion are of size $12^{3} \times 28$ with lattice spacing $a \sim 0.20$ $\mathrm{fm}$, lattice size $L a=2.4 \mathrm{fm}(\beta=6.0$ and $\kappa=0.1530)$, and $N_{\text {conf }}=300$. The pion masses are approximately 175, 186, 197, 208, 223, 242, 264, 293, 327, 359, 391, 426, 466, 512, 566, 625, 681, 717, 752, 813, 899, 961, 1006, 1152, 1291, and $1547 \mathrm{MeV}$. Only results with successful extraction of first excited states are shown in this paper.

In general meson results contain much more noise than baryon results for both the ground and first excited states. Fig. 2 shows the results for pions and $K^{*}$. The results for the last several lightest quarks in the pion case are not reliable enough to be included. In the $K^{*}$ case, we can see clearly the trend of how the data points approach the physical point.

We obtained better results with baryons than with mesons. Fig. 3,4,5 show the results for $\Lambda, \Sigma$, $\Xi, \Delta, N, N^{\prime}$, and $N^{*}$ Again, aside from $N^{\prime}$ and $N^{*}$, all data points approach the physical points very well. In the case of $\Xi$ in Fig. 4, the MEM data points approach $2.0 \mathrm{GeV}$ value which is a possible indication of $\Xi(1950)$.

Fig. 5 shows that the masses of $N^{*}(1535)$ are slightly higher than those of the Roper resonance $N^{\prime}(1440)$ for lighter quark masses $\left(0.4<m_{\pi}<0.6 \mathrm{GeV}\right)$ which is consistent with observed spectra. However, this order is switched where the masses of $N^{*}(1535)$ become lower than those of $N^{\prime}(1440)$ for heavier quark masses in $0.6<m_{\pi}<1.0 \mathrm{GeV}$ region. This is not only consistent with a previous study of excited nucleons in Ref. [3] about level ordering of these two states, but it also clarifies their level ordering. 

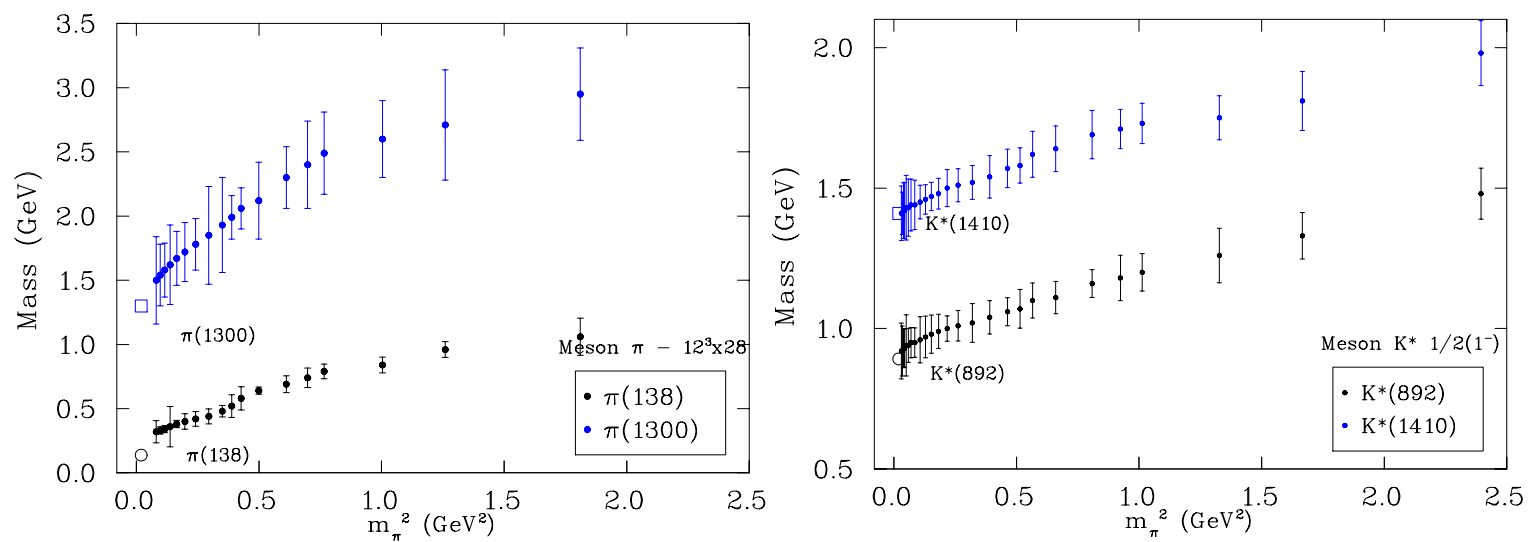

Figure 2: Ground and first excited states of pion and $K *$ as a function of the square of the pion mass in the physical unit. The experimental values are marked by empty symbols.
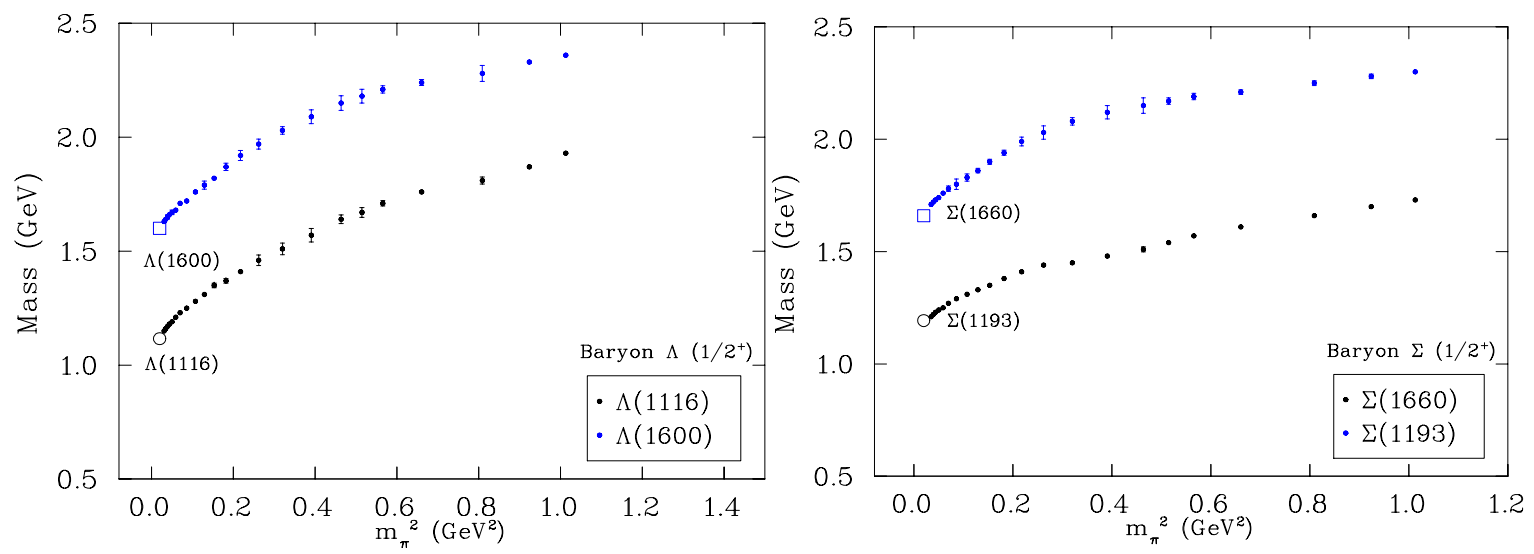

Figure 3: Ground and first excited states of Octet $\Lambda$ and Octet $\Sigma$
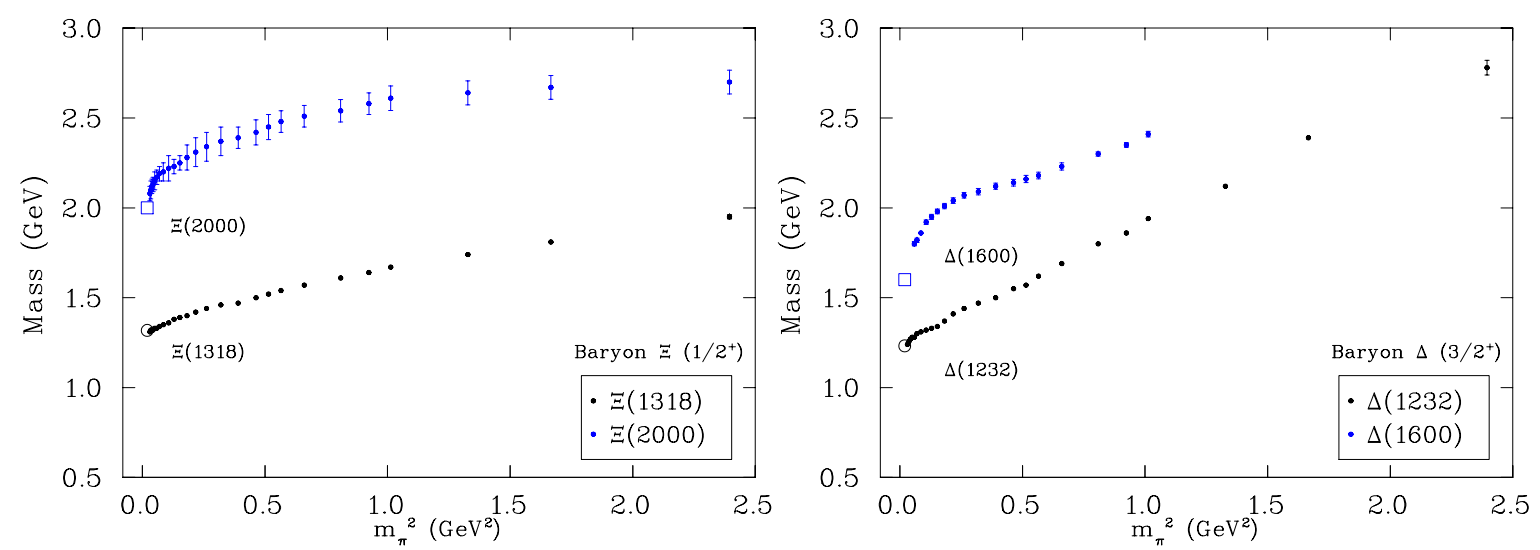

Figure 4: Ground and first excited states of Octet $\Xi$ and Decuplet $\Delta$ 


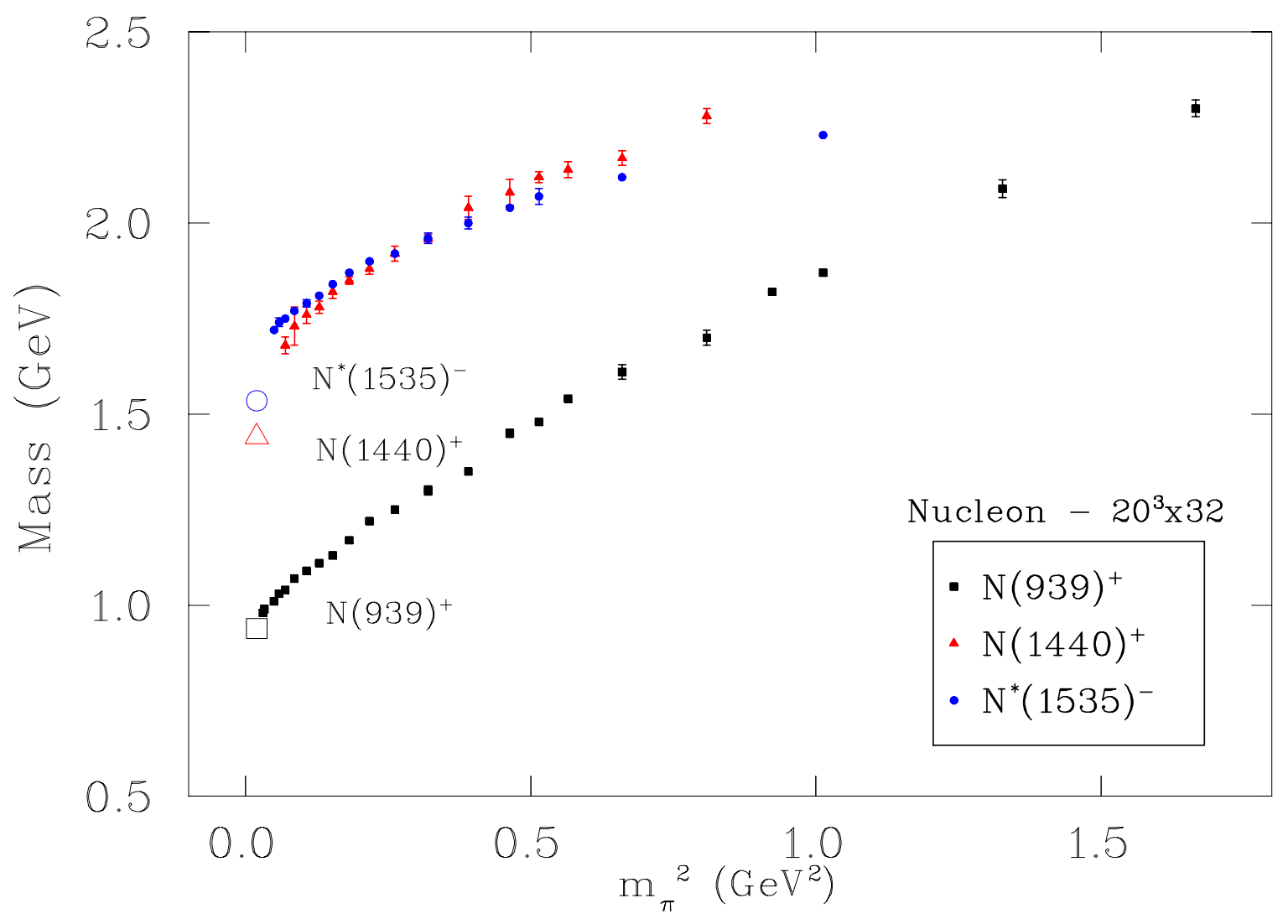

Figure 5: Nucleon.

\section{Conclusion}

We tested and used MEM for mock data and lattice QCD data. MEM is shown to be able to extract and study the ground and first excited states of some light hadrons in quenched LQCD with some success. In this study factors that limit MEM ability to produce SPF is noise and lattice spacing. We have not done chiral extrapolation with a curve fit to the physical point since the results are not refined enough. However, the data points approach their respective physical points quite well. Greater number of configurations of correlation functions with finer lattices and better actions that reduce more noise are needed to perform the chiral extrapolation reliably.

\section{Acknowledgment}

This work is supported in part by U.S. Department of Energy under grant DE-FG02-95ER40907.

\section{References}

[1] Y. Nakahara, M. Asakawa, T Hatsuda, Phys. Rev. D 60, 091503 (1999), arXiv:hep-lat/9905034.

[2] M. Asakawa, T. Hatsuda, Y. Nakahara, Prog. Part. Nucl. Phys. 46, 459 (2001), arXiv:hep-lat/0011040.

[3] K. Sasaki, S. Sasaki, T. Hatsuda, Phys. Lett. B 623, 208 (2005), arXiv:hep-lat/0504020. 
[4] R.K. Bryan, Eur. Biophys. J. 18, 165 (1990).

[5] T. Harsono, Ph.D. thesis in preparation.

[6] H. Neuberger, Phys. Lett. B 417, 141 (1998).

[7] $\chi$ QCD Collaboration, http://eagle.phys.gwu.edu/fxlee/chiQCD.html; N. Mathur, Y. Chen, S.J. Dong, T. Draper, I. HorvÃąth, F.X. Lee, K.F. Liu, J.B. Zhang, Phys. Lett. B605, 137 (2005);

S.J. Dong, F.X. Lee, K.F. Liu, and J.B. Zhang, Phys. Rev. Lett. 85, 5051 (2000). 